\title{
Cluster-Exact Approximation of Spin Glass Groundstates
}

\author{
Alexander K Hartmann * \\ Institut für Theoretische Physik \\ Ruprecht-Karls-Universität Heidelberg \\ Philosophenweg 19 \\ 69120 Heidelberg, Germany
}

June 21, 2018

PACS: 75.10.H, 75.10.N, 02.10

\begin{abstract}
We present an algorithm which calculates groundstates of Ising spin glasses approximately. It works by randomly selecting clusters of spins which exhibit no frustrations. The spins which were not selected, contribute to the local fields of the selected spins. For the spin-cluster a groundstate is exactly calaculated by using graphtheoretical methods. The other spins remain unchanged. This procedure is repeated many times resulting in a state with low energy. The total time complexity of this scheme is approximately cubic. We estimate that the groundstate energy density of the infinite system for the $\pm J$ model is $-1.400 \pm 0.005$ (2d) and $-1.766 \pm 0.002(3 \mathrm{~d})$. The distribution of overlaps for selected systems is calculated in order to characterize the algorithm.
\end{abstract}

The combination of frustration and randomness makes it difficult to find groundstates of spin glasses [1] using numerical simulations. In the past years many methods [2] have been proposed including the multicanonical ensemble [3], genetic algorithms [4], a scheme, which uses storing of spin configurations [5], and an exact algorithm exhibiting exponential timecomplexity [6]. In this paper

*e-mail: hartmann@lattice.tphys.uni-heidelberg.de 
we present a method which combines clustering of spins with exact calculation of groundstates of polynomial solvable problems in order to calculate approximately groundstates of Edwards-Anderson (EA) spin glasses.

The Hamiltonion of the EA model is given by

$$
H=-\sum_{<i j>} J_{i j} \sigma_{i} \sigma_{j}-\sum_{i} B_{i} \sigma_{i}
$$

where the sum goes over nearest neighbours of spins $\sigma_{i}= \pm 1$. The exchange interactions $J_{i j}$ are selected at random according to a probability distribution. The external field $B_{i}$ can be site-dependend.

For our computer experiments we used simple cubic lattices of $N=L^{d}$ spins ( $L=$ linear lenght, $d=$ dimension) with periodic boundary conditions in no external field $\left(B_{i}=0\right)$. The interactions are $J_{i j}= \pm 1$ with equal probability using the constraint $\sum_{<i j>} J_{i j}=0$.

The algorithm for the approximation of groundstates in frustrated Ising systems works by taking a spin configuration and calculating another one, which has a lower or equal energy. This procedure is iterated many times.

The idea of the scheme for lowering the energy is to choose a cluster of spins, which exhibits no frustrations. The interaction of the cluster with the spins at its boundary is included into the local fields of the cluster-spins. The groundstate of the cluster is exactly calculated by using concepts of graph theory [7, 8, 9]: An equivalent network is constructed [10], the maximum flow is calculated with the Ford-Fulkerson algorithm [11] and a minimum cut is constructed [12]. The new configuration consists of the unchanged spins, which are not in the cluster, and the groundstate of the cluster. By definition this procedure cannot increase the energy.

For describing the spin-cluster the Hamiltonian can be rewritten in the form

$$
H_{c}=-\sum_{<i j>} J_{i j} t_{i} t_{j} \sigma_{i}^{c} \sigma_{j}^{c}-\sum_{i} B_{i}^{c} t_{i} \sigma_{i}^{c}+C
$$

The values of $t_{i}=0, \pm 1$ describe the cluster and are used to handle antiferromagnetic interactions. If spin $\sigma_{i}=: t_{i} \sigma_{i}^{c}$ does not belong to the cluster: $t_{i}=0$. If $t_{i} \neq 0$ and $t_{j} \neq 0$ their signs have to be choosen so that $J_{i j}^{c}:=J_{i j} t_{i} t_{j}>0$, because only Hamiltonians with positive exchange interactions can be transformed into an equivalent network. The local fields $B_{i}^{c}$ include the external field $B_{i}$ and the interactions with the neighbours of spin $\sigma_{i}$, which are not in the cluster:

$$
B_{i}^{c}=B_{i}+\sum_{<j>} J_{i j}\left(1-\left|t_{j}\right|\right) \sigma_{j}
$$

The constant $C$ summarizes the interactions between the non-cluster spins and can be dropped. The construction of the unfrustrated cluster works in the following way: a spin is randomly selected as seed. Iteratively neighbouring spins are added, if no frustration occours. If a cluster cannot be extended, one 
more seed-spin is selected. The following algorithm contains the details. The local variables $\delta_{i}$ are used to indicate the spins, which are already detected as neighbours of the cluster. The set $A$ contains the neighbours of spin $\sigma_{i}$, which are already in a cluster, whereas $B$ contains the neighbours, which are added to the boundary of the cluster.

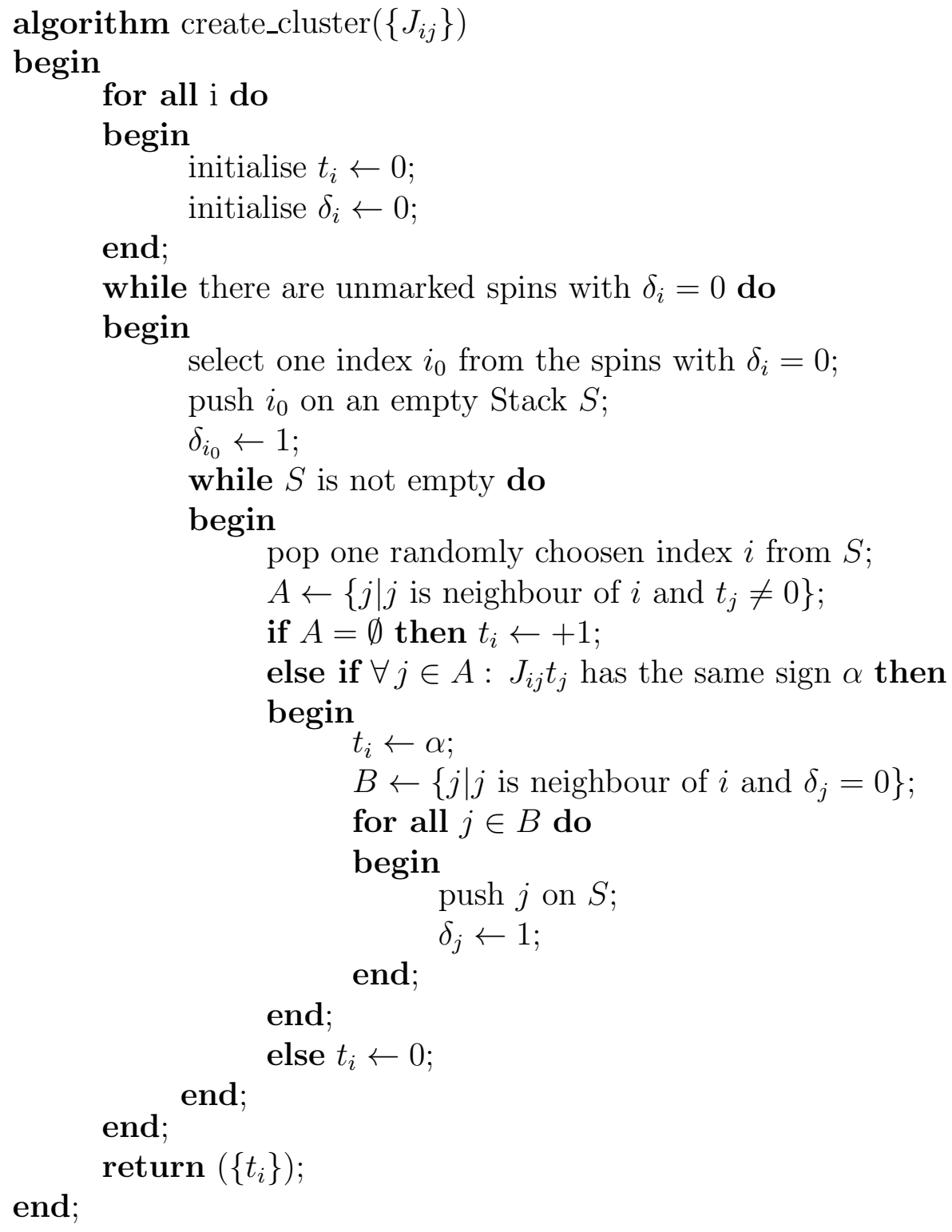

A run for calculating a groundstate consists of choosing randomly an initial configuration or choosing all spins pointing up, and calculating new configurations until the energy can not further be lowered. For this in our experiments we found as a good criterium that the energy did not change for the last $n_{g}$ steps. We used $n_{g}=N / 2(2 \mathrm{~d}, 3 \mathrm{~d})$ as rule of thumb, because in some very long runs we observed, that the longest period between two jumps in energy never 
exceeded this value and scaled almost linear with system size.

Because the minimum energy reached in a run depends on the starting configuration and on the clusters, which were constructed, we performed several runs for each realization of the random variables $J_{i j}$. We used that one, which ended on the lowest energy level. We found 3 runs per realization sufficent, because by increasing the number of runs, the average groundstate energy was only lowered about 0.01 percent.

Because in each step the spin-cluster is randomly built and by using the algorithm [12] all of the degenerated cluster-groundstates have a positive probability to be calculated, usually in each step a new spin configuration is constructed, even if the energy remains constant. So it is possible to explore large areas of the configuration space.

In figure 11 the average energy density $e_{L}(t)=E_{L}(t) / N$ of 96 realizations is shown as a function of the step number for lattice sizes $L=4,6,16$ of the $2 \mathrm{~d}$ system. One can see, that in the beginning the algorithm approaches very fast low values. Later the decrease in energy is very small.

It is possible to use other procedures in selecting the spins of the cluster. We tried three other methods (we call the first presented algorithm method $A$ ):

method B Not only neighbours of cluster-spins were added to the stack $S$, but neighbours of all spins, which were tested, if they can be added to the cluster.

method C The spins which were tested, if they can be added to the cluster were totaly selected at random from the spins, which were yet untested.

method D Methods A and B are applied alternately.

We tested all four methods by calculating groundstates for eight realizations of $16^{2}$ systems (3 runs each). In table 1 the average groundstate energy density $e^{0}$ is displayed. Also we constructed for the $2 \mathrm{~d}$ and $3 \mathrm{~d}$ case 100 respectively 10 spin-clusters for 100 realizations of system sizes $L=4,6, \ldots, 20$. The average size fractions $n_{c} / N$ of the spin-clusters are shown in the last two rows.

\begin{tabular}{r|cccc}
\hline method & $\mathrm{A}$ & $\mathrm{B}$ & $\mathrm{C}$ & $\mathrm{D}$ \\
\hline$e^{0}$ & 1.425 & 1.425 & 1.415 & 1.427 \\
$n_{c} / N(2 \mathrm{~d})$ & 0.708 & 0.696 & 0.644 & - \\
$n_{c} / N(3 \mathrm{~d})$ & 0.578 & 0.568 & 0.541 & - \\
\hline
\end{tabular}

Table 1: Comparison of four methods used to construct spin-clusters

We observed, that for some realizations method A and for others method B gave lower energies, so it is plausible, that the combination of both methods 
gave the best results. So we used method D for all further experiments. Maybe better methods for constructing the cluster would result in a faster convergence and/or lower energies.

To estimate the groundstate energies of the $2 \mathrm{~d}$ and $3 \mathrm{~d} \pm J$ spin glass we performed calculations for lattice sizes $L=4, \ldots, 30(2 \mathrm{~d})$ and $L=4, \ldots, 14$ (3d) with $96\left(4^{2}, \ldots, 20^{2}, 4^{3}, \ldots, 10^{3}\right)$, respectively $32\left(30^{2}, 12^{3}, 14^{3}\right)$ realizations of the random variables $J_{i j}$.

In figure 2 the groundstate energy density $e^{0}(L)$ of the $2 \mathrm{~d}$ systems is plotted as function of system size. To estimate the groundstate energy density of the infinite system we performed a finite-size scaling analysis. A fit to the function $f_{L}^{0}(N)=f_{\infty}^{0}+c / N$ [3] results in $e^{0}=-1.400 \pm 0.005$. This value is consistent with earlier results from a genetic algorithm $-1.400 \pm 0.005$ [4], multicanonical simulations $-1.394 \pm 0.007$ [3] pure MC $-1.407 \pm 0.008$ [13] and transfer matrix calculations $-1.4024 \pm 0.0012$ [14].

The results for the $3 \mathrm{~d}$ systems are shown in figure 3. The fit gives a groundstate energy density of $e^{0}=-1.766 \pm 0.002$. The results of other authors are $1.7863 \pm$ 0.0028 [3] and $1.765 \pm 0.01$ [4]

As seen in figure 1 the time for approaching the groundstate increases rapidly with the system size. The average number of steps needed to calculate a groundstate are shown in figure 1 as a function of the number of spins $N$. For the $2 \mathrm{~d}$ system (lower curve) a fit to a function $t(N)=a+b N^{c}$ results in a timecomplexity of $O\left(n^{1.17 \pm 0.06}\right)$. The result for the $3 \mathrm{~d}$ system is similiar: $O\left(n^{1.27 \pm 0.08}\right)$. Because the time to calculate one groundstate of the cluster increases quadraticly with the number of spins [12] and the size of the cluster is linear in the system size, this totally results in an approximate cubic timecomplexity of the algorithm. Performing one run for example for a $10^{3}$ system consisting of 1000 configurations needed about 10 hours on a $80 \mathrm{Mhz}$ PowerPC processor system. In order to characterize the way the algorithm approaches the groundstates we selected one realization of a $2 \mathrm{~d}(L=16)$ system and used configurations of different energy densities $e_{s} \in[-1.414,1.0]$ as starting-configurations. For each configuration 8 different runs for constructing groundstates were performed. For each run, which resulted in the groundstate energy density value of $e^{0}=-1.414$ the last 100 configurations were stored. The overlap

$$
q:=\sum_{i=1}^{N} \sigma_{i}^{\alpha} \sigma_{i}^{\beta}
$$

was calculated between all pairs $(\alpha, \beta)$ of groundstates belonging to a startingconfiguration. We obtained the corresponding probability distributions $P_{L}(q)$ by counting the numbers of overlaps within intervals of length $\Delta q=0.05$. The result is displayed in figure 5 for the starting energies $e_{s}=-1.414,-1.07,0.117$. For these three staring energies 800, 300 respectively 400 of the resulting states exhibited the lowest energy density. One can see, that the algorithm approaches 
local minima of the energy landscape. The higher the starting energy the more minima are reachable. If a system is caught in a local minimum, only a restricted area of configuration space can be explored by the algorithm, although in each step a different configuration is generated which is indicated by $P_{L}(1)=0$. But for other realizations we got also different distributions. There are systems where $P_{L}(q)$ is always narrow or always broad, independent of the starting energy. The accessible area is usually smaller for lower groundstate energies than for higher lying groundstates.

In conclusion, in this letter we have presented an algorithm for the approximation of groundstates for Ising spin glasses. Its time complexity is approximately cubic in the number of spins. Because by each step a large number of spins is allowed to flip, the algorithm approaches low energies very rapidly.

So it should be interesting to try combinations with other algorithms like genetic algorithms or Monte Carlo simulations, or to use it for other Ising type optimization problems.

Although we applied it to the $\pm J$ model it is also possible to treat more general forms of lattices, interactions and their probability distributions.

It should possible to get results for $B \neq 0$ in a resonable amount of time in order to characterize better the phase diagramm along the $T=0$ axis.

\section{Acknowledgments}

We would like to thank D W Heermann, K D Usadel and G Reinelt for fruitfull discussions, A Linke for a critical reading of the manuscript and the Paderborn Center of Parallel Computing for the allocation of computer time on a PARIXPowerPC System. 


\section{References}

[1] For reviews see

Binder K and Young A P, Rev. Mod. Phys. 58, 801-976 (1986);

Fisher K H and Hertz J A, Spin Glasses, Cambridge University Press (1991)

[2] Rieger H, in Annual Reviews of Computational Physics II, ed. Stauffer D, World Scientific, Singapore (1995)

[3] Berg B A and Celik T, Phys. Rev. Lett. 69, 2292-2295 (1992);

Berg B A, Celik T and Hansmann U, Europhys. Lett. 22, 63-68 (1993);

Hansmann U H E and Berg B A, Int. J. Mod. Phys. C 5, 263-265 (1994)

[4] Sutton P, Hunter D L and Jan N, J. Physique I 4, 1281-1285 (1994)

[5] Krauth W and Pluchery O, J. Phys. A 27, L715-L720 (1994)

[6] Barahona F, Phys. Rev. B 49, 12864-12867 (1994)

[7] Claiborne J D, Mathematical preliminaries for computer networking, John Wiley \& Sons, New York 1990

[8] Knödel W, Graphentheoretische Methoden und ihre Anwendung, Springer, Berlin 1969

[9] Tarjan R E, Data Structures and Network Algorithms, Society for Industrial and Applied Mathematics, Philadelphia, 1983

[10] Picard J P and Ratliff H D, Networks 5, 357-370 (1975)

[11] Ford L R and Fulkerson D R, Canadian J. Math. 8, 399-404 (1956)

[12] Hartmann A K and Usadel K D, Physica A 214, 141-152 (1995)

[13] Wang J-S and Swendsen R H, Phys. Rev. B 38, 4840-4844 (1988)

[14] Cheung H-F and McMillan W L, J. Phys C 16, 7027-7032 (1983) 


\section{Figure Captions}

Figure 11 Average energy density $e_{L}(t)=E_{L}(t) / N$ of $2 \mathrm{~d} \pm J$ spin glass at different iterations $t$. Shown are 3 sizes $L=4,6,16$.

Figure 2 Average groundstate energy density $e^{0}(L)=E^{0}(L) / N$ of $2 \mathrm{~d} \pm J$ spin glass as a function of size $L$.

Figure 3 Average groundstate energy density $e^{0}(L)=E^{0}(L) / N$ of $3 \mathrm{~d} \pm J$ spin glass as a function of size $L$.

Figure 1 Average number of steps needed to reach a groundstate as function of system size $N$ for 2 d system $(L=4, \ldots, 20)$.

Figure 5 Distribution of overlaps for one single realization $\left(2 \mathrm{~d}, L=16, e^{0}=\right.$ -1.414). For different starting configurations with energies $e_{s}=0.117$, $-1.07,-1.414$ with 8 independent runs 100 groundstates were calculated. The states with energy density $e^{0}=-1.414$ were included in the calculation of the distribution, i.e. 400, 300, 800 states for the three values of $e_{s}$. The lines are guides for the eyes only. 


\section{Figures}

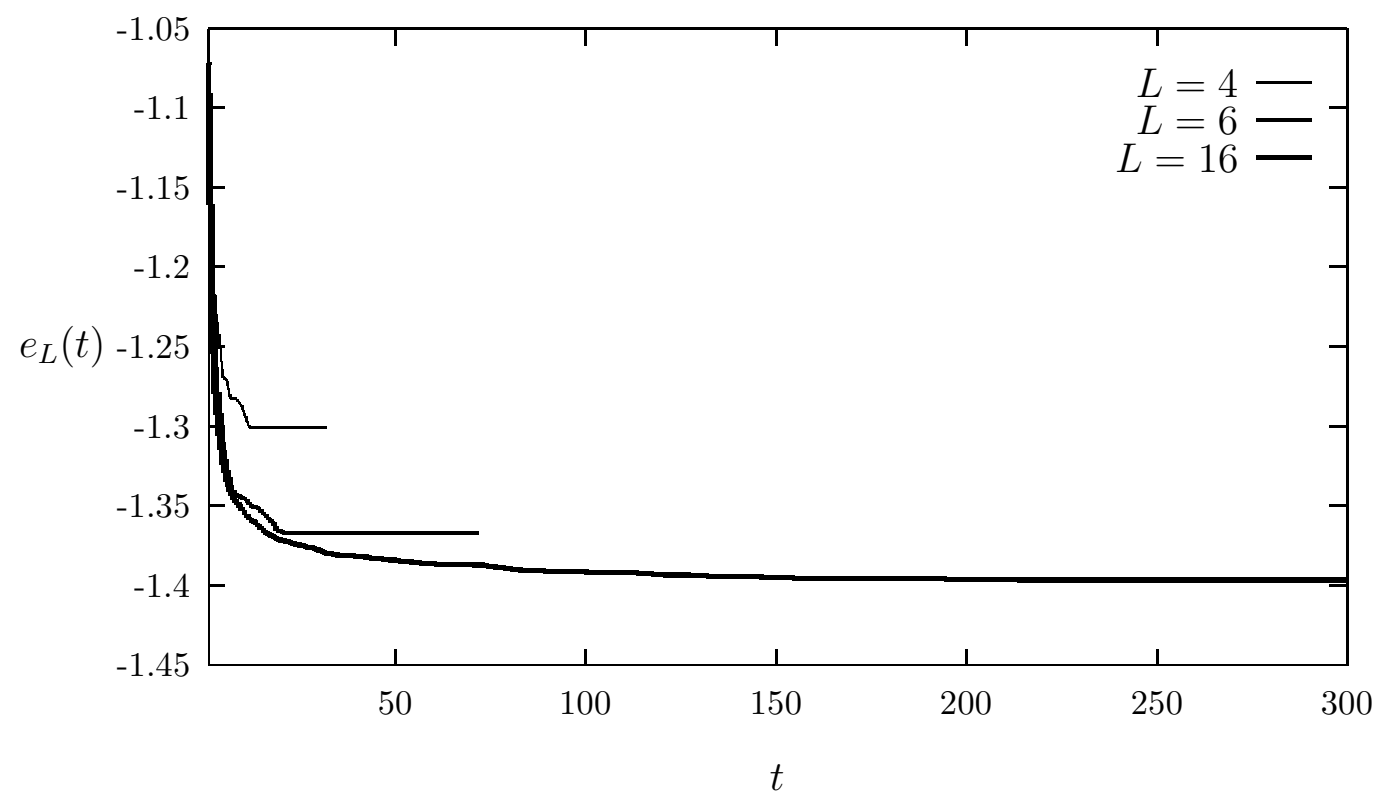




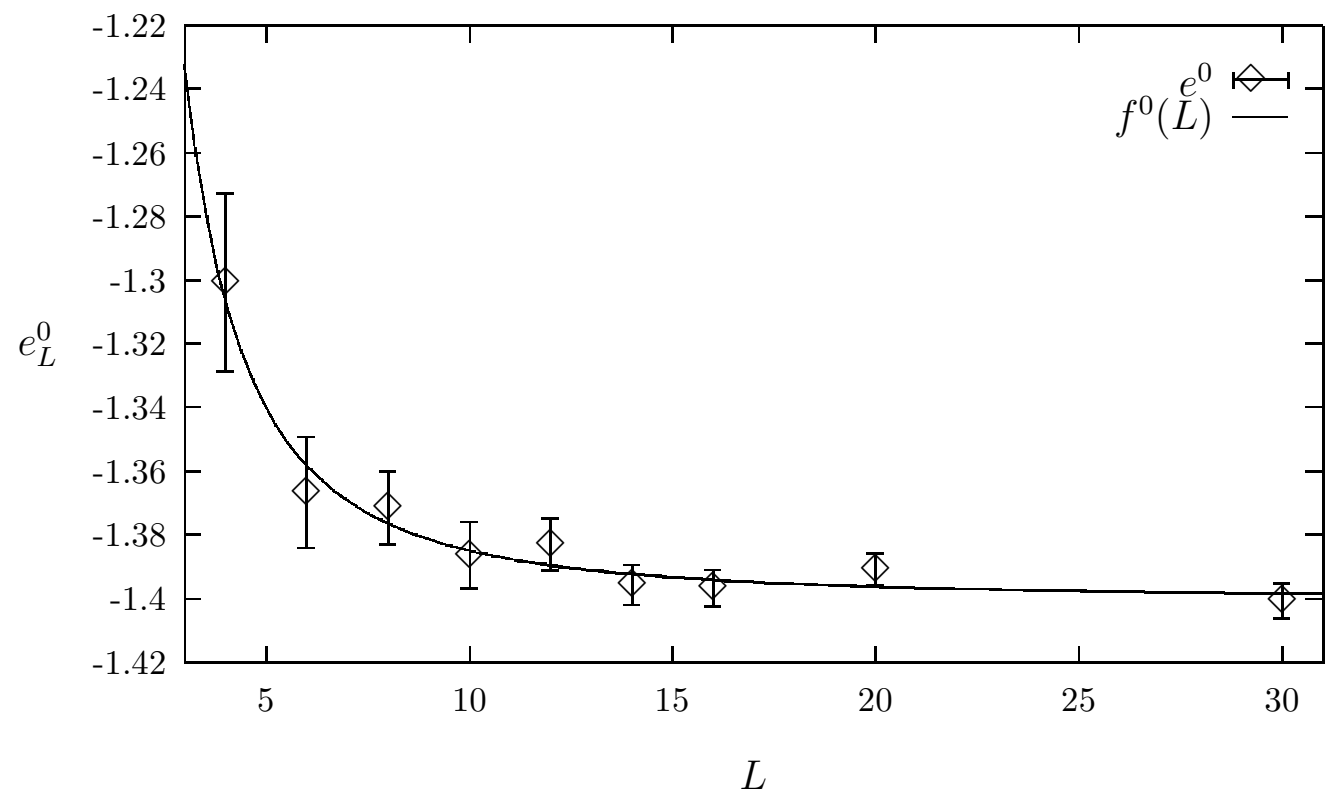




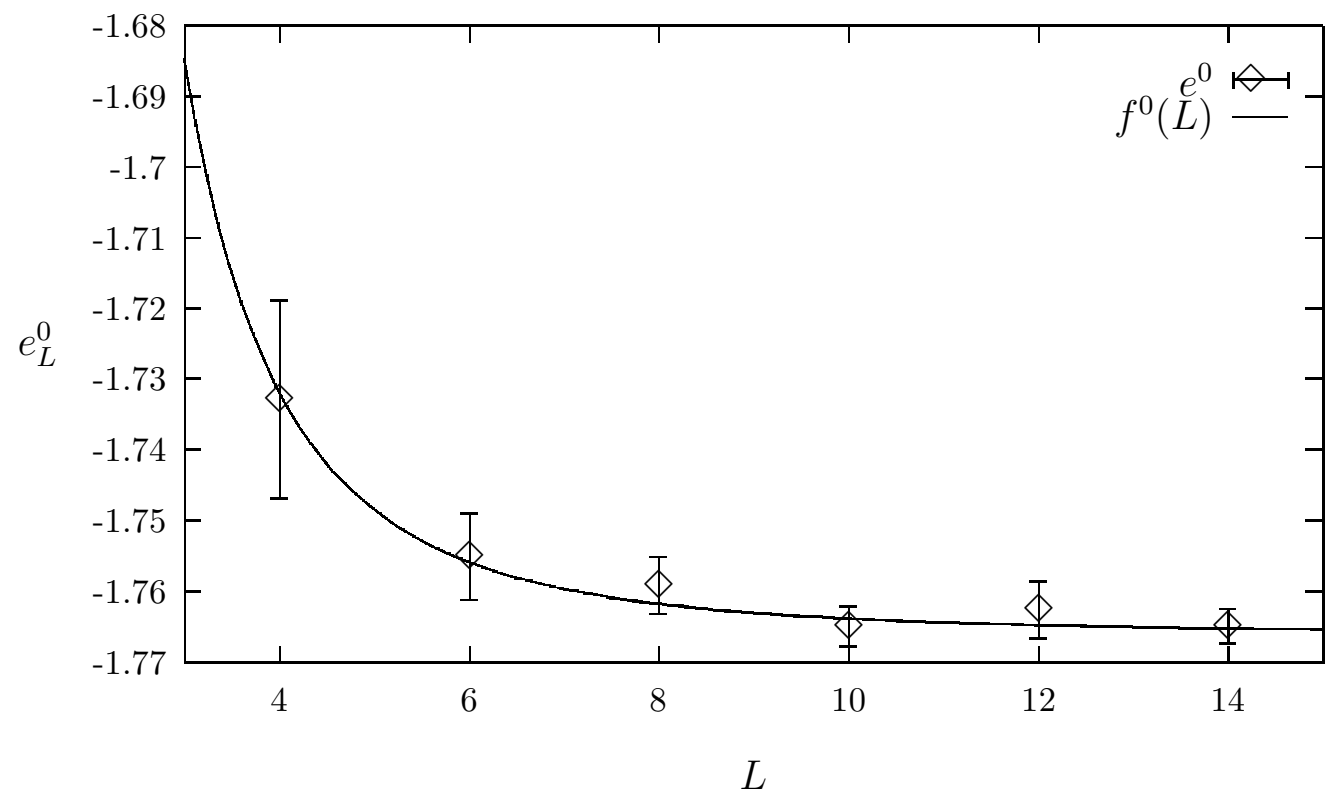




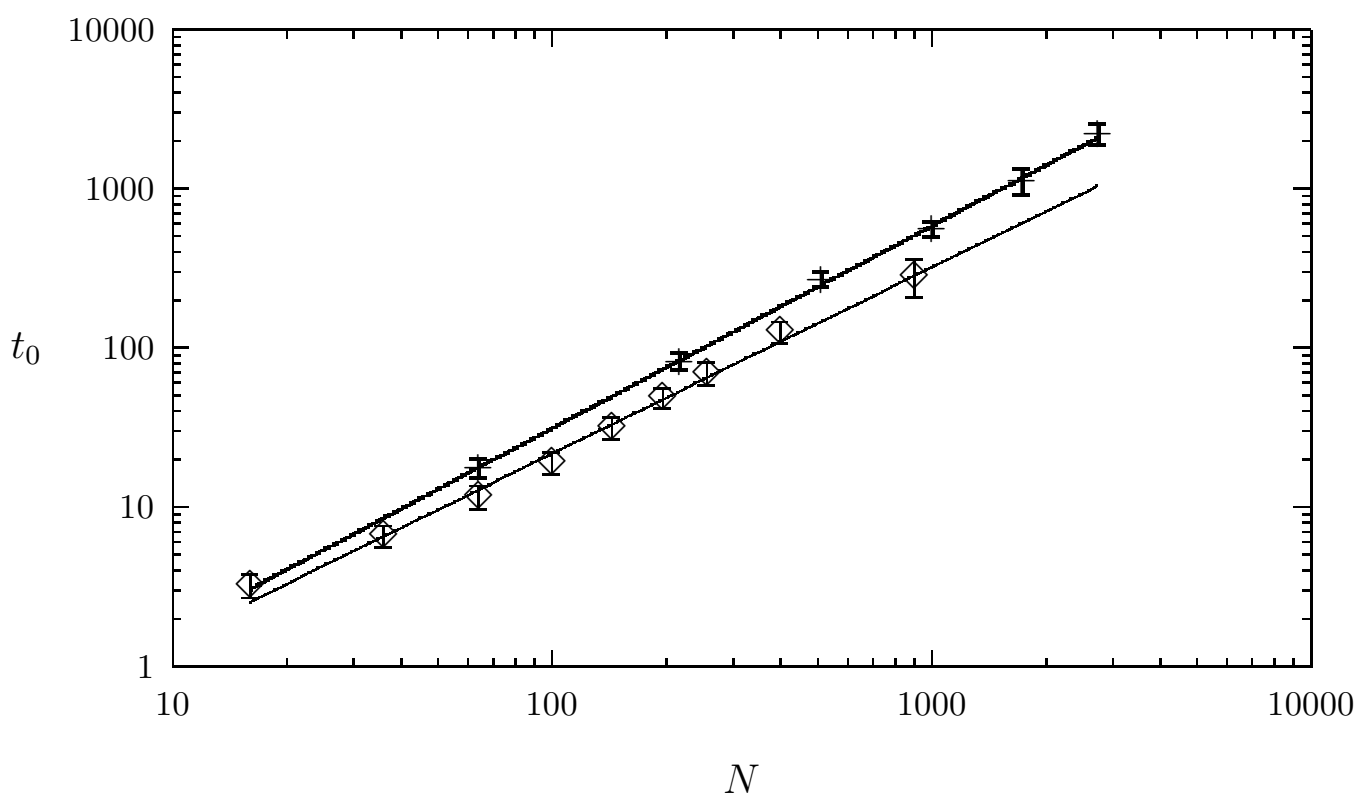




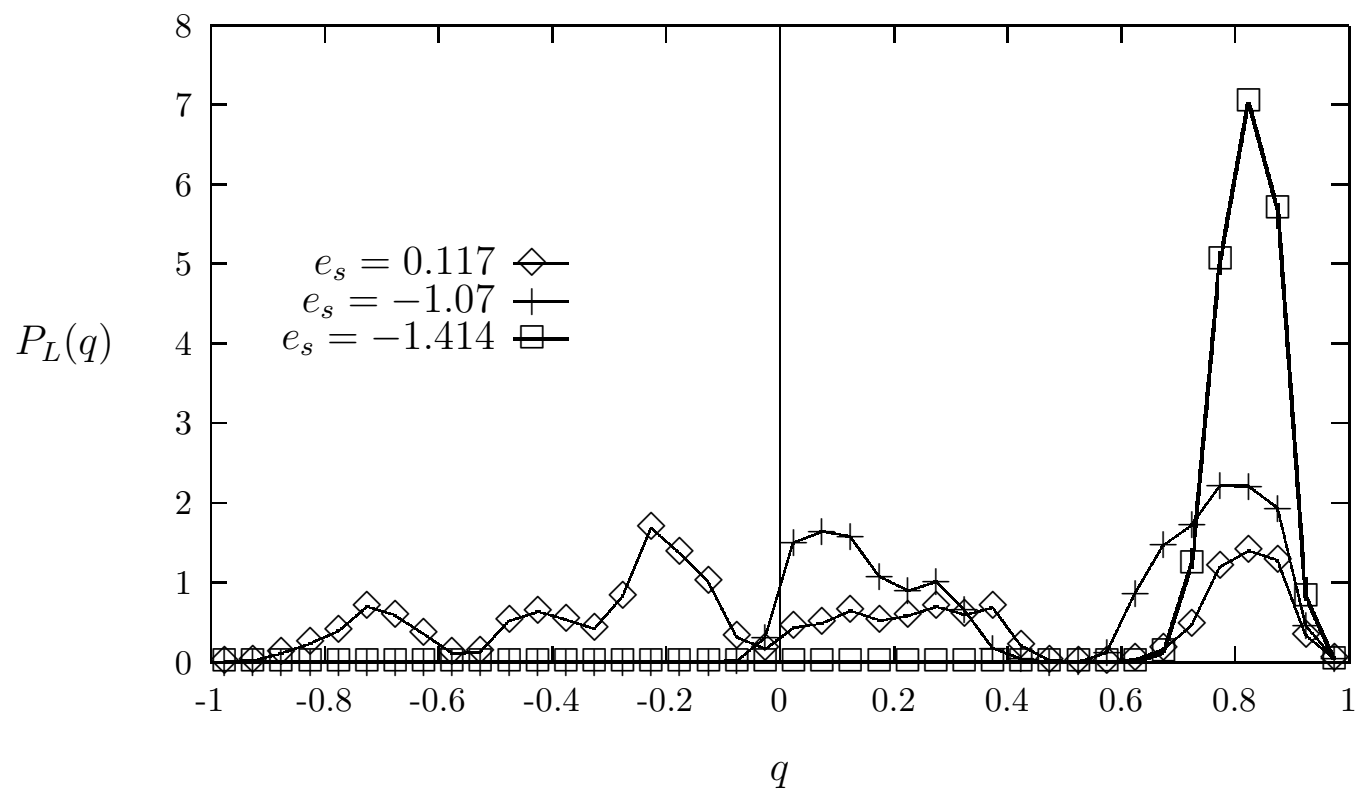

